\title{
O Desenvolvimento da Consciência Morfológica nos Estágios Iniciais da Alfabetização
}

\section{Development of Morphological Awareness in the Initial Stages of Literacy}

\author{
Márcia Maria Peruzzi Elia da Mota ${ }^{*}, a$, Anne-Sophie Besse ${ }^{b}$, Jaqueline Dias $^{c}$, \\ Nádia Paiva ${ }^{c}$, Stella Mansur-Lisboa ${ }^{c} \&$ Danielle Andrade Silva $^{c}$ \\ ${ }^{a}$ Universidade do Estado do Rio de Janeiro, Rio de Janeiro, Brasil, ${ }^{b}$ Université Rennes 2, Paris, França \\ $\&{ }^{c}$ Universidade Federal de Juiz de Fora, Juiz de Fora, Brasil
}

\begin{abstract}
Resumo
Evidências de estudos recentes mostram que a consciência morfológica está relacionada à aquisição da leitura e da escrita. Entretanto, a idade da aquisição dessa habilidade ainda é uma questão controversa. Identifica-se na literatura dois grupos que tratam desse desenvolvimento: teóricos que defendem que essa aquisição é tardia e os que defendem que ela acontece desde os estágios iniciais da alfabetização e interage com a escolarização. Apresentamos a primeira parte de um estudo longitudinal que observou o desenvolvimento da consciência morfológica no português do Brasil. Cinco tarefas foram usadas avaliar a consciência morfológica de crianças de 6 a 9 anos de idade. Os resultados mostram que essa habilidade interage com a escolarização desde os anos iniciais da alfabetização.

Palavras-chave: Consciência morfológica; Consciência metalingüística; Morfologia; Desenvolvimento metalingüístico.
\end{abstract}

\begin{abstract}
Evidence from recent studies has shown that morphological awareness relates to both reading and spelling acquisition. However, the age of acquisition of this ability is still a matter of controversy. Literature review indentifies two groups of researchers: those that think that this development starts late and those for whom it starts early and interacts with schooling. This paper is the first part of a longitudinal study which looks at the development of morphological awareness in Brazilian Portuguese. Five tasks are used to evaluate 6 to 9 year-old children's morphological awareness. The results show that this ability interacts with schooling at an early age.

Keywords: Morphological awareness; Metalinguistic awareness; Morphology; Metalinguistic development.
\end{abstract}

Nas últimas três décadas observamos uma profusão de estudos que investigaram a relação entre a consciência metalingüística e a alfabetização (Gombert, 1992; Mota, 2009). Dentre as habilidades metalingüísticas duas se destacam por contribuirem de forma causal para o sucesso na alfabetização: a consciência fonológica e a morfoló-

\footnotetext{
* Endereço para correspondência: Universidade do Estado do Rio de Janeiro, Instituto de Psicologia, Campus Francisco Negrão de Lima, Pavilhão João Lyra Filho, R. São Francisco Xavier, 524, $10^{\circ}$ andar, Bloco B, Sala 10.019, Maracanã, Rio de Janeiro, RJ, Brasil, CEP 20550-900. E-mail: mmotapsi@gmail.com

Agradecimentos ao financiamento do Conselho Nacional de Desenvolvimento Científico e Tecnológico (CNPq) Edital Ministério da Ciência e Tecnologia (MCT)/CNPq 50/2006 - Ciências Humanas, Sociais e Sociais Aplicadas.

Bolsista de Iniciação Científica do CNPq Stella Mansur-Lisboa; bolsista de iniciação científica da Fundação de Amparo à Pesquisa do Estado de Minas Gerais (FAPEMIG) Jaqueline Dias Comitê de ética: (protocolo Comitê de Ética em Pesquisa (CEP)/Universidade Federal de Juiz de Fora (UFJF) 956002 2007)
}

gica. Dessas duas, a mais estudada é a primeira, que pode ser definida como a habilidade de refletir sobre os sons que compõem a fala (Cardoso-Martins, 1995). Estudos demonstram que a consciência fonológica ajuda na alfabetização e que o treinamento dessa habilidade auxilia na remediação dos problemas de leitura (Capovilla \& Capovilla, 2009, para uma revisão). No Brasil, os trabalhos de Capovilla e Capovilla (2000), Cardoso-Martins (1995), Guimarães (2003), Maluf e Barrera (1997), e Santos (2003), entre outros, confirmam a importância do tema para aquisição da língua escrita no português brasileiro.

No entanto, mais recentemente, o interesse dos pesquisadores têm se voltado para o papel facilitador de outra habilidade metalingüística para alfabetização: a consciência morfológica (Carlisle, 1988, 1995, 2000; Colé, 2004; Colé, Royer, Leuwers, \& Casalis, 2004; Roman, Kirby, Parrila, Wade-Woolley, \& Deacon 2009). A habilidade de refletir sobre os morfemas, que são as menores unidades lingüísticas com significado próprio, é chamada de consciência morfológica (Carlisle, 1995) e pode 
ajudar na alfabetização porque a ortografia de muitas palavras depende da morfologia da língua. Palavras como "laranjeira", por exemplo, que têm ortografia ambígua, podem ser escritas de forma correta se soubermos sua origem: "laranja". Igualmente, seus significados podem ser inferidos através da leitura se soubermos o significado da palavra de origem.

Embora muitos estudos tenham demonstrado a importância da consciência morfológica para alfabetização em diferentes ortografias (Abu-Rabia, 2007; Boukadida, Gombert, \& Maaouia, 2009, em árabe; Casalis \& Louis Alexandre, 2000; Colé, 2004, em francês; Levin, Ravid, \& Rapaport, 2001, em hebraico; Mota, Annibal, \& Lima, 2008; Mota \& Silva, 2007, em português), o seu desenvolvimento ainda é uma questão controversa. Pesquisadores interessados no desenvolvimento da consciência morfológica se polarizam em dois grupos. O primeiro considera que a consciência morfológica se desenvolve tardiamente, depois de alguns anos de escolarização formal (Nagy, Berninger, \& Abbot, 2006; Nunes \& Bryant, 2006). O segundo sustenta que a consciência morfológica começa a se desenvolver cedo e interage com a escrita desde os estágios iniciais (Colé, Marec-Breton, Royer, \& Gombert, 2003).

Em um estudo longitudinal, Nunes, Bryant e Bindman (1997) mostraram que as crianças parecem passar por uma seqüência de estágios no entendimento de como as palavras são formadas. Os autores, que estudaram a aquisição da escrita de morfemas como o "ed" no inglês, argumentam que antes que as crianças possam escrever palavras morfologicamente complexas, que fogem aos padrões de correspondência entre letra e som do inglês, elas precisam ter entendido o princípio alfabético.

O resultado do estudo de Nunes et al. (1997) está de acordo também com os principais modelos de desenvolvimento da escrita que trabalham com a idéia de desenvolvimento em estágios e de que o processamento das palavras em morfemas ocorre após a aquisição do princípio alfabético (Frith, 1985; Marsh, Friedman, Welsh, \& Desberg, 1980).

No entanto, é possível que o desenvolvimento da escrita possa não ocorrer em estágios e que também não seja necessário que as crianças tenham adquirido o princípio alfabético antes de usar informações morfológicas. Estudos posteriores ao de Nunes et al. (1997) têm mostrado que as crianças se beneficiam da morfologia da língua desde cedo no processo de alfabetização. Isso nos leva ao segundo grupo: o de teóricos que consideram que o processamento da morfologia ocorre desde cedo.

Colé et al. (2003) estudaram crianças francesas nos anos iniciais de aprendizagem da leitura. Nesse estudo. as crianças tinham que ler quatro grupos de palavras: (a) morfologicamente complexas (ex., 'banheiro' 'banho'+'eiro'); (b) com a mesma seqüência de letras, mas morfologicamente simples (ex., 'dinheiro'); (c) pseudopalavras morfologicamente complexas (ex., 'linheiro' - 'linho'+'eiro'); (d) pseudopalavras não sufixadas (ex., 'binheiro').

Apesar de as palavras no estudo de Colé et al. (2003) terem as mesmas características fonológicas e número de letras, as crianças cometeram menos erros lendo as palavras do grupo A acima do que as do grupo B. Estes resultados indicam um efeito facilitador da estrutura morfológica no reconhecimento de palavras desde os anos iniciais de escolarização.

No Brasil, Mota (2008a) solicitou que crianças de segundo e de terceiro ano resolvessem seis tarefas de consciência morfológica. Cinco envolviam julgamento da respostas corretas e uma era de produção. Dentre as tarefas, duas envolviam o julgamento da raiz de palavras prefixadas e sufixadas. As crianças deviam decidir qual dentre duas palavras era da mesma família que uma palavra chave (e.g. Cansar - Descanso ou Desmaio). As outras duas tarefas seguiam as mesmas instruções. Desta vez solicitavam que o participante decidisse sobre o significado de afixos em palavras sufixadas e prefixadas (e.g: "Descobrir" é feita da mesma forma que "Deslizar" ou "Desfazer"). Além dessas duas tarefas, as crianças tinham que fazer um julgamento sobre a relação morfológica entre dois pares de palavras ("chique" é da mesma família que "chiqueiro"?) e uma tarefa de analogia gramatical baseada na tarefa de Nunes et al. (1997). Essa última envolvia a produção de uma resposta correta.

Os resultados apontaram que, para as quatro primeiras tarefas de julgamento, as crianças de segundo ano atingiram níveis de acerto bem acima do nível de chance. Para as tarefas de julgamento da relação semântica entre palavras e para a de produção os níveis de acerto foram de $63 \%$ para associação e $52 \%$ para analogia gramatical.

Os resultados de Mota (2008a) indicam que crianças com dois anos de escolarização formal são capazes de fazer julgamentos sobre a morfologia da língua. Mesmo nas tarefas de produção as crianças foram capazes de produzir respostas corretas em pelo menos metade dos itens de produção. Quando a tarefa era simplificada, o índice de acerto encontrou-se acima do nível de chance.

O papel do tipo de tarefa no resultado de pesquisas que investigam o desenvolvimento morfossintático está bem documentado na literatura (Correa, 2005; Gombert, 1992; Mota, 1996). Diferentes tarefas exigem graus de controle cognitivo diversos. As de julgamento são mais fáceis, pois todas as respostas possíveis estão presentes. A criança apenas tem que julgar qual delas é a resposta correta. Alguns autores consideram que esse tipo de tarefa envolve um conhecimento mais implícito do processamento morfossintático (Gombert, 1992). No caso do estudo de Mota (2008a), havia diferenças entre as tarefas de julgamento. As que envolviam julgamento dos afixos reduziam as pistas semânticas apresentadas na raiz das palavras, por isso poderia ser considerada mais implícita.

No entanto, definir processamento implícito e explícito em Psicologia é uma questão controversa. Gombert 
(1992) argumenta que algum grau de habilidade metalingüística é necessário para se aprender a ler. Esse grau inicial é chamado por ele de epilingüístico e envolve um conhecimento implícito da língua. Ao longo do processo de alfabetização, o contato com a língua escrita tornaria o conhecimento metalingüístico mais explícito.

Não é do escopo desse estudo esclarecer a distinção entre conhecimento explícito e implícito em psicologia. Porém, é nosso objetivo estabelecer que grau de conhecimento da morfologia da língua as crianças apresentam ao começar seu processo de alfabetização e como este interage com a alfabetização. Essa questão traz implicações importantes, pois como citado anteriormente, estudos têm demonstrado que o conhecimento morfossintático ajuda no processo de alfabetização em várias ortografias alfabéticas (Belajouza, 2003, em árabe; Ben-Dror, Bentin, \& Frost, 1995, em hebraico; Carlisle \& Nomanbhoy, 1993, em inglês; Casalis \& Louis-Alexandre, 2000, em francês; Müller \& Brady, 2001, em finlandês). No português, evidências obtidas com crianças que já se alfabetizaram corroboram esses resultados (Mota et al., 2008; Mota \& Silva, 2007). Menos se sabe sobre o desenvolvimento dessa habilidade no início do ensino formal da língua escrita.

Este estudo faz parte de um estudo maior que investigou o desenvolvimento da consciência morfológica nos primeiros anos de alfabetização em crianças falantes do português Os escores em cinco tarefas de consciência morfológica, com diferentes graus de complexidade são comparados para os três primeiros anos de alfabetização formal. Se crianças nos estágios iniciais da alfabetização são capazes de processar a língua escrita, espera-se que as crianças acertem mais da metade dos itens na tarefa de produção. Espera-se, também, que haja uma progressão com a série escolar no desenvolvimento da consciência morfológica e que as tarefas de julgamento sejam mais fáceis do que as de produção.

\section{Método}

Os participantes da presente pesquisa fazem parte de um estudo longitudinal que investiga a relação entre a consciência morfológica e a alfabetização. A amostra consistiu de 71 crianças, sendo 29 alunas do $1^{\circ}$ ano, 19 alunas do $2^{\circ}$ ano e 23 do $3^{\circ}$ ano, todas do ensino fundamental de escolas particulares, situadas no interior de Minas Gerais. A média de idade das crianças de primeiro ano era 76, 6 meses $(D P=7,1)$, de segundo ano, 87,5 meses $(D P=3,93)$ e de terceiro ano 98,3 meses $(D P=4,58)$. O convite para participar foi feito através de uma carta convite, em que se solicitava a autorização do responsável através do Termo de Consentimento Livre e Esclarecido.

\section{Instrumentos}

Tarefas de Consciência Morfológica

Tarefas de Decisão Morfológica - Raiz (Uma variação de Besse, 2009, e Vidigal de Paula, Gombert, \& da Silva
Leme, 2009). As tarefas de Besse (2009) e de Vidigal de Paula et al. (2009) foram inicialmente delineadas para crianças mais velhas do que as que participaram desse estudo. Simplificamos a tarefa original, criando uma variação da mesma que dessa vez focalizava a raiz das palavras e não os afixos. Utilizou-se o termo variação e não adaptação, pois esta se refere a um conjunto de procedimentos psicométricos que se adota para adequar um teste a um contexto diferente do que foi criado. Nesta tarefa a criança deveria decidir qual palavra seria da mesma família que a palavra alvo.

Os pares de palavra foram escolhidos em razão do número de letras e da freqüência de ocorrência na escrita, todas elas extraídas da tabela para primeira série proposta por Pinheiro (1996). Como não há índices de familiaridade para o português, as palavras foram pareadas por freqüência para garantir minimamente um equilíbrio. A lista consistia de dez grupos de três palavras envolvendo prefixos (Ex: Tornar - Retorna - Resolve) e dez grupos de palavra envolvendo sufixos (Ex. Pinta - Tambor - Pintor). As crianças poderiam obter um total de 10 pontos nessa análise.

Tarefa de Decisão Morfológica (Besse, 2009; Vidigal de Paula et al., 2009). Nesta tarefa esperava-se que o participante julgasse se uma palavra alvo era construída da mesma forma que outras duas palavras. A lista consistia de 12 grupos de três palavras envolvendo prefixos (e.g., Descobrir - Deslizar, Desfazer) e 12 grupos de palavra envolvendo sufixos (e.g., Chaveiro-Pandeiro-Cinzeiro). As crianças poderiam obter um total de 12 pontos nessa tarefa.

Tarefa de Analogia Gramatical (adaptada de Nunes et al., 1997). A tarefa inicial de Nunes et al. (1997) foi adaptada, considerando a especificidade da morfologia derivacional do português. Sob essa perspectiva, foram criados dez itens, a partir dos quais as crianças deveriam produzir uma palavra morfologicamente complexa a partir de uma palavra alvo, aplicando a mesma relação de derivação de um par previamente dado. O total de pontos possíveis era 10 .

\section{Procedimento}

A avaliação da consciência morfológica das crianças foi feita em seções individuais, e os testes de consciência morfológica foram aplicados tal como descrito a seguir. Nas tarefas de consciência morfológica intituladas "Decisão Morfológica - raiz" foi dada a seguinte instrução: "algumas palavrinhas são da mesma família do que outras. Por exemplo, a palavra "conta" e a palavra "reconta" são da mesma família. Já a palavra "bola" e "rebola" não são da mesma família. Eu vou falar para você uma palavra e depois vou falar mais outras duas e você vai me dizer qual das duas é da mesma família da primeira". Por fim, ofereceu-se um exemplo para a criança: "a palavra "gela" é da mesma família que "congela" ou "conversa"? Quando a criança errava, explicava-se a forma correta, e se acertasse, a tarefa era iniciada. Depois do exemplo, 
Mota, M. M. P. E., Besse, A-S., Dias, J., Paiva, N., Mansur-Lisboa, S. \& Silva, D. A. (2011). O Desenvolvimento da Consciência Morfológica nos Estágios Iniciais da Alfabetização.

começava-se a testagem, mesmo que a criança não conseguisse acertar.

A seguir, aplicou-se a Tarefa de Decisão Morfológica (Besse, 2009; Vidigal de Paula et al., 2009), dando-se a seguinte explicação à criança: "em português há palavras que são da mesma família, como, por exemplo, "descobrir" e "cobrir", ou seja, "descobrir" vem de "cobrir". Para fazer uma outra palavra acrescenta-se uma pequena coisa no início. Outro exemplo é o caso de "desfazer" e "fazer", onde acrescenta o "des" no início de "fazer". Porém, há palavras que também se iniciam por "des", mas não vêm de outra palavra. Depois dessas considerações, voltou-se a pedir para a criança "então qual a palavrinha que é feita da mesma maneira que "descobrir"? É “deslizar" ou é "desfazer?". Se a criança respondesse corretamente, iniciava-se a tarefa; do contrário, oferecia-se a forma correta explicando a razão. Depois do exemplo, iniciou-se a testagem mesmo quando a criança não conseguiu acertar.

Por último, aplicou-se a Tarefa de Analogia Gramatical (adaptada de Nunes et al., 1997) com a instrução de que muitas palavras poderiam ser relacionadas. A aplicadora apresentou um par de palavras relacionadas e pediu à criança que, depois de ouvir uma palavra, criasse uma outra relacionada como no exemplo. A tarefa era iniciada sempre pelo exemplo: "pedra-pedreiro; leite- ?" e assim, sucessivamente, eram pronunciadas as demais palavras-alvo.

\section{Resultados}

A Tabela 1 mostra a média e o desvio padrão para cada tarefa de consciência morfológica por série. As tarefas de julgamento envolvendo a raiz das palavras (decisão morfológica envolvendo a raiz) foram as mais fáceis para as crianças. O nível de chance aqui é de $50 \%$. A média de acertos em nível de chance demonstraria que as crianças não estão processando a morfologia ao fazer julgamentos sobre as palavras. Respostas correta poderiam ocorrer por acaso. A Tabela 1 apresenta a média e o desvio padrão para as tarefas de consciência morfológica. No primeiro ano, as crianças acertaram um pouco mais de $80 \%$ das respostas tanto para sufixos como para os prefixos na tarefa de julgamento com base na raiz (decisão morfológica envolvendo a raiz). Para a tarefa de julgamento baseado nos afixos a porcentagem de acerto foi de 62\% (decisão morfológica - Besse, 2009; Vidigal de Paula et al., 2009), acima do nível de chance. Para os participantes de outros anos, os resultados foram próximos do teto.

Para a tarefa de analogia não foi possível calcular o nível de chance, pois as crianças não puderam escolher aleatoriamente as respostas corretas. Essa tarefa era, portanto, uma medida mais conservadora da habilidade das crianças refletirem sobre a morfologia da língua, as crianças do primeiro ano acertaram mais da metade dos itens $(55 \%$ de acerto).

Tabela 1

Média e Desvio Padrão para as Tarefas de Consciência Morfológica

\begin{tabular}{lcccccc}
\hline Ano Escolar & & $\begin{array}{c}\text { Raiz prefixo } \\
\mathrm{N}=10 \text { itens }\end{array}$ & $\begin{array}{c}\text { Raiz sufixo } \\
\mathrm{N}=10 \text { itens }\end{array}$ & $\begin{array}{c}\text { Prefixos } \\
\mathrm{N}=12 \text { itens }\end{array}$ & $\begin{array}{c}\text { Sufixos } \\
\mathrm{N}=12 \text { itens }\end{array}$ & $\begin{array}{c}\text { Analogia } \\
\mathrm{N}=10 \text { itens }\end{array}$ \\
\hline Primeiro ano & Média & 8,24 & 8,34 & 7,51 & 7,55 & 5,51 \\
$N=29$ & $D P$ & 1,96 & 1,79 & 2,14 & 2,81 & 2,41 \\
Segundo ano & Média & 9,63 & 9,47 & 6,36 & 6,36 & 6,00 \\
$N=19$ & $D P$ & 0,59 & 0,69 & 1,97 & 2,19 & 1,88 \\
Terceiro ano & Média & 9,73 & 9,65 & 6,34 & 6,00 & 7,56 \\
$N=23$ & $D P$ & 0,61 & 0,71 & 2,14 & 1,80 & 1,64 \\
Total & Média & 9,09 & 9,07 & 6,83 & 6,73 & 6,30 \\
$N=71$ & $D P$ & 1,50 & 1,39 & 2,15 & 2,43 & 2,21 \\
\hline
\end{tabular}

A análise das médias mostra uma tendência à melhoria do processamento morfológico para as tarefas de julgamento baseados na raiz (decisão morfológica envolvendo a raiz) e para a de analogia, mas não para as de julgamento com base nos afixos (decisão morfológica - Besse, 2009; Vidigal de Paula et al., 2009). Nessas as crianças de segundo e terceiro ano tiveram desempenho inferior aos das crianças de primeiro ano.

Para verificar se as diferenças encontradas entre séries foi significativa ou resultado de variações ao acaso, cinco análises de variâncias univariadas foram realizadas. A variável independente era série, com três níveis (primei- ro, segundo e terceiro ano) e a variável dependente o número de acertos em cada tarefa. Os resultados mostram resultados significativos para a tarefa de decisão morfológica baseada na raiz de palavras sufixadas $\left(F_{(2,68)}=\right.$ $10,05, p<0,0001)$, para a tarefa de decisão-baseada na raiz de palavras prefixadas $\left(F_{(2,68)}=8,04, p<0,001\right) \mathrm{e}$ para a tarefa de analogia $\left(F_{(2,68)}=6,67, p<0,002\right)$.

A análise das médias usando como teste post hoc $t$ de Student mostrou que, para tarefa de decisão morfológica baseada na raiz de palavras prefixadas, as crianças de primeiro ano tiveram desempenho inferior aos das crianças de segundo ano e terceiro ano. As crianças de segun- 
do e terceiro ano não diferiram entre si $(t(46)=-2,99$ e $p$ $<0,004$ para comparação entre primeiro ano e segundo ano e $t(50)=-3,53$ e $p<0,001$ para comparação entre primeiro ano e terceiro ano). Para tarefa de decisão morfológica baseada na raiz de palavras sufixadas os resultados foram similares. As crianças de primeiro ano apresentaram desempenho inferior aos das crianças de segundo ano e terceiro ano $(t(46)=-2,6$ e $p<0,01$ para comparação entre primeiro ano e segundo ano e $t(50)=$ 3,28 e $p<0,02$ para comparação entre primeiro ano e terceiro ano). Não houve diferenças entre os grupos.

Para a tarefa de analogia não houve diferenças entre o primeiro e segundo ano. Porém, as crianças de terceiro ano obtiveram resultados significativamente superiores aos das crianças do primeiro e segundo ano $(t(50)=-3,47$, $p<0,01$ para comparações entre o primeiro e o terceiro ano, e $t(40)=-2,87, p<0,007$ para comparações entre o segundo e o terceiro ano).

Já a tarefa de julgamento baseada no afixo de palavras sufixadas não apresentou diferenças significativas entre as séries $\left(F_{(2.68)}=3,07, p=0,08\right)$. O mesmo ocorreu para a tarefa de julgamento baseada no afixo de palavras prefixadas $\left(F_{(2,68)}=2,60, p=0,053\right)$.

\section{Discussão}

O presente trabalho visou investigar o desenvolvimento da consciência morfológica em crianças falantes do português do Brasil. As crianças no primeiro ano da alfabetização acertaram um pouco mais de $80 \%$ das respostas nas tarefas de julgamento baseadas na raiz tanto para os sufixos quanto para prefixos. Para as séries posteriores os resultados chegaram perto do máximo de acerto (efeito de teto). O nível de chance nessa tarefa é de $50 \%$. Os resultados demonstram então que, desde cedo, quando pistas semânticas são disponíveis, as crianças podem reconhecer a relação entre as palavras. Sugere-se, então, que o processamento morfológico não depende da experiência com alfabetização, mas interage com ela.

Uma nota de caução deve ser dada, no entanto, em relação a esses resultados. As palavras nas listas apresentadas na tarefa de julgamento baseada na raiz das palavras eram muito semelhantes em termos fonológicos. É possível que o julgamento das crianças possa ter sido baseado na semelhança fonológica das palavras e não apenas em fatores morfológicos. É importante demonstrar que os julgamentos feitos pelas crianças foram devido a semelhanças morfológicas e não fonológicas. As outras três tarefas podem lançar luz sobre essa questão.

As duas tarefas de julgamento que reduziam as pistas semânticas e fonológicas foram mais difíceis. Ainda assim, a média geral de acerto nessa tarefa foi de $67 \%$, acima do nível de chance. É interessante notar que essa tarefa não resultou em diferenças entre as séries. Mota (2008b) mostrou que essas duas tarefas apresentam baixos coeficientes confiabilidade (alpha de Cronbach $<0,07)$. Outra explicação possível é a de que o desenvol- vimento do processamento dos afixos se desenvolve tardiamente. Contudo, em conjunto, esses resultados mostram que as crianças de primeiro ano, média de idade 76,6 meses, quando têm as demandas das tarefas reduzidas, são capazes de processar a morfologia da língua.

Assim, é possível argumentar que diferentes tarefas exigem níveis de controle diversos sobre a morfologia da língua. Tarefas que exigiam decisões mais explícitas sobre a morfologia da língua, como a de analogia gramatical, servem como uma medida mais conservadora do conhecimento da estrutura morfológica. A atuação das crianças nessa tarefa foi de $55 \%$ corroborando as conclusões apresentadas no início dessa discussão.

Os dados apresentam resultados interessantes em termos de um modelo de desenvolvimento. Para as tarefas de julgamento com base na raiz não houve diferenças entre o desempenho das crianças de segundo ano e o terceiro ano, mas as crianças do primeiro ano tiveram desempenho inferior. Para a tarefa de analogia, que envolvia um maior controle da estrutura da língua, o terceiro ano apresentou desempenho superior que os outros dois anos. Podemos interpretar esses resultados como sugerindo um padrão no desenvolvimento. As crianças de primeiro ano mostraram desempenho inferior aos da terceira em todas as tarefas. Contudo, as crianças de segundo ano apresentaram desempenho semelhante às das de terceiro ano nas tarefas que exigiam julgamentos menos profundos, mas quando grau de dificuldade da tarefa era maior, seu desempenho se assemelhava às de primeiro ano. Por fim, as crianças de terceiro ano tiveram desempenho superior em todas as tarefas.

$\mathrm{Na}$ introdução argumentamos que pesquisadores interessados no desenvolvimento da consciência morfológica se polarizam em dois grupos. O primeiro considera que a consciência morfológica se desenvolve tardiamente, depois de alguns anos de escolarização formal (Nagy et al., 2006; Nunes \& Bryant, 2006). O segundo defende que a consciência morfológica começa a se desenvolver cedo e interage com a escrita desde os estágios iniciais (Colé et al., 2003). Nossos resultados são consistentes com um modelo interativo, como o segundo. As crianças de primeiro ano têm um conhecimento, ainda que rudimentar, da morfologia da língua, mas à medida que vão se escolarizando esse conhecimento se aprofunda. As crianças de segundo ano parecem estar em um estágio intermediário, em relação às crianças de primeiro e terceiro ano. Por fim, as crianças de terceiro ano apresentaram um maior conhecimento da estrutura morfológica das palavras.

Estudos futuros devem testar esse modelo de desenvolvimento através de estudos longitudinais e com um escopo grande de tarefas. Os estudos longitudinais permitiriam a avaliação do desenvolvimento dessas habilidades controlando para as diferenças individuais.

As implicações educacionais também devem ser pensadas. Uma vez que se possa estabelecer um modelo do desenvolvimento da consciência morfológica no portu- 
Mota, M. M. P. E., Besse, A-S., Dias, J., Paiva, N., Mansur-Lisboa, S. \& Silva, D. A. (2011). O Desenvolvimento da Consciência Morfológica nos Estágios Iniciais da Alfabetização.

guês do Brasil e sua relação com o desenvolvimento da escrita, podemos pensar em estudos de intervenção, que ao desenvolver essas habilidades poderão melhorar o desempenho na leitura e na escrita.

\section{Referências}

Abu-Rabia, S. (2007). The role of morphology and short vowelization in reading Arabic among normal and dyslexic readers in grades 3, 6, 9, and 12. Journal of Psycholinguist Research, 36, 89-106.

Belajouza, M. (2003). Stratégies d'identification des mots en arabe, compétences phonologiques et morphologiques des lecteurs en difficulté. In M. N. Romdhane, J.-E. Gombert, \& M. Belajouza, L'apprentissage de la lecture. Perspectives comparatives: Collection Psychologie (pp. 195-211). Rennes, France: Presses Universitaires de Rennes.

Ben-Dror, I., Bentin, S., \& Frost, R. (1995). Semantic, phonologic, and morphologic skills in reading disabled and normal children: Evidence from perception and production of spoken Hebrew. Reading Research Quarterly, 30(4), 876893.

Besse, A. S. (2009). Caractéristiques des langues premières et apprentissage de la lecture en français langue seconde: perspective comparative interlangue entre l'arabe et le portugais. In N. Marec-Breton, A. S. Besse, F. de la Haye, N. BonnetonBotté, \& E. Bonjour (Eds.), L'apprentissage de la langue écrite: approche cognitive (pp. 133-150). Rennes, France: Presses Universitaires de Rennes.

Boukadida, N., Gombert, J. E., \& Maaouia, A. (2009). Contribution des conaissaces morphologiques à l'apprentissage de la lecture en arabe. In N. Marec-Breton, A. S. Besse, F. de la Haye, N. Bonneton-Botté, \& E. Bonjour (Eds.), L'apprentissage de la langue écrite: approche cognitive (pp. 107-118). Rennes, France: Presses Universitaires de Rennes.

Capovilla, A., \& Capovilla, F. (2000). Efeitos do treino de consciência fonológica em crianças com baixo nível sócio-econômico. Psicologia: Reflexão e Crítica, 13(1), 7-24.

Capovilla, A., \& Capovilla, F. (2009). A consciência fonológica e sua importância para a aquisição da linguagem escrita. In M. Mota (Ed.), Desenvolvimento metalinguístico: Questões contemporâneas (pp. 19-40). São Paulo, SP: Casa do Psicólogo.

Cardoso-Martins, C. (Ed.). (1995). Consciência fonológica e alfabetização. Petrópolis, RJ: Vozes.

Carlisle, J. (1988). Knowledge of derivational morphology and spelling ability in fourth, six, and eight graders. Applied Psycholinguistics, 9, 247-266.

Carlisle, J. (1995). Morphological awareness and early reading achievement. In L. Feldman (Ed.), Morphological aspects of language processing (pp. 189-211). Hillsdale, NJ: Lawrence Erlbaum.

Carlisle, J. (2000). Awareness of the structure and meaning of morphologically complex words: Impact on reading. Reading and Writing: An Interdisciplinary Journal, 12, 169-190.

Carlisle, J. F., \& Nomanbhoy, D. M. (1993). Phonological and morphological awareness in first graders. Applied Psycholinguistics, 14(2), 177-195.

Casalis, S., \& Louis-Alexandre, M.-F. (2000). Morphological analysis, phonological analysis and learning to read french: A longitudinal study. Reading and Writing, 12(3), 303-335.
Colé, P. (2004). Le traitement des mots morphologiquement complexes au cours de l'acquisition de la lecture: des données préliminaires. In L. Ferrand, J. Grainger, \& J. Segui (Eds.), Psycholinguistique cognitive (pp. 307-328). Bruxelles, Belgique: De Boeck Université.

Colé, P., Marec-Breton, N., Royer, C., \& Gombert, J. (2003). Morphologie des mots et apprentissage de la lecture. Reeducation Orthophonic, 213, 57-60.

Colé, P., Royer, C., Leuwers C., \& Casalis, S. (2004). Les connaissances morphologiques dérivationnelles et l'apprentissage de la lecture chez l'apprenti-lecteur français du CP au CE2. L'Année Psychologique, 104(4), 701-750.

Correa, J. (2005). A avaliação da consciência morfossintática na criança. Psicologia: Reflexão e Crítica, 18(1), 91-97.

Frith, U. (1985). Beneath the surface of developmetal dislexia. In K. Patterson, M. Coltheart, \& J. Marshal (Eds.), Surface dislexia (pp. 301-330). London: Lawrence Erlbaum.

Gombert, J. (1992). Metalinguistic development. Hertfordshire, UK: Harverster Whesheaf.

Guimarães, S. (2003). Dificuldades no desenvolvimento da lectoescrita: O papel das habilidades metalinguísticas. Psicologia: Teoria e Pesquisa, 19(1), 33-45.

Levin, I., Ravid, D., \& Rapaport, S. (2001). Morphology and spelling among Hebrew-speaking children: From kindergarten to first grade. Journal of Child Language, 28(3), 741-772.

Maluf, M. R., \& Barrera, S. (1997). Consciência fonológica e linguagem escrita em pré-escolares. Psicologia: Reflexão e Crítica, 10(1), 125-145.

Marsh, G., Friedman, M., Welsh, V., \& Desberg, P. (1980). The development of strategies in spelling. In U. Frith (Ed.), Cognitive processes in spelling (pp. 339-353). London: Academic Press.

Mota, M. (1996). Children's role of grammatical rules in spelling. Unpublished doctoral dissertation, Oxford University, UK.

Mota, M. (2008a). Algumas considerações a respeito do que as crianças sabem sobre a morfologia derivacional. Interação (Curitiba), 12(1), 115-123.

Mota, M. (2008b). Avaliação da consciência da morfologia derivacional: Fidedignidade e validade. Avaliação Psicológica, 7(2), 151-157.

Mota, M. (Ed.). (2009). Desenvolvimento metalingüístico: Questões contemporâneas. São Paulo, SP: Casa do Psicólogo.

Mota, M., Annibal, L., \& Lima, S. (2008). A morfologia derivacional contribui para a leitura e escrita no português? Psicologia: Reflexão e Crítica, 21(2), 311-318.

Mota, M., \& Silva, K. (2007). Consciência morfológica e desenvolvimento ortográfico: Um estudo exploratório. Psicologia em Pesquisa, 1(2), 86-92.

Müller, K., \& Brady, S. (2001). Correlates of early reading performance in a transparent orthography. Reading and Writing: An Interdisciplinary Journal, 14, 757-799.

Nagy, W., Berninger, V., \& Abbot, R. (2006). Contributions of morphology beyond phonology to literacy outcome of upper elementary and middle-school students. Journal of Educational Psychology, 98(1), 134-147.

Nunes, T., Bryant, P., \& Bindman, M. (1997). Morphological spelling strategies: Developmental stages and processes. Developmental Psychology, 33(4), 637-649.

Nunes, T., \& Bryant, P. (2006). Improving literacy by teaching morphemes. London: Routledge. 
Pinheiro, A. M. V. (1996). Contagem de freqüencia de ocorrência de palavras expostas a crianças na faixa pré-escolar e séries iniciais do $1^{\circ}$ grau [Computer software]. São Paulo, SP: Associação Brasileira de Dislexia.

Roman, A. A., Kirby, J. R., Parrila, R. K., Wade-Woolley, L., \& Deacon, S. H. (2009). Toward a comprehensive views of the skills involved in word reading in Grades 4, 6, and 8. Journal of Experimental Child Psychology, 102, 96-113.

Santos, A. (2003). A influência da consciência fonológica na aquisição da leitura e escrita. In F. Sisto, G. Oliveira, Fini, L., M. T. de Souza, \& R. Brenelli (Eds.), Atuação psicopedagógica em aprendizagem escolar. Petrópolis, RJ: Vozes.

Vidigal de Paula, F., Gombert, J. E., \& da Silva Leme, I. (2009). Acquisition de la conscience morpho-dérivationnelle, de la lecture et de l'orthographe, chez des enfants brésiliens, de la première à la septième année de scolarité. In N. Marec-Breton, A. S. Besse, F. de la Haye, N. Bonneton-Botté, \& E. Bonjour (Eds.), L'apprentissage de la langue écrite: approche cognitive (pp. 119-131). Rennes, France: Presses Universitaires de Rennes. 